\title{
Twelve months of follow-up after intravitreal injection of ranibizumab for the treatment of idiopathic parafoveal telangiectasia
}

This article was published in the following Dove Press journal:

Clinical Ophthalmology

4 July 2013

Number of times this article has been viewed

\author{
Alexandros Rouvas \\ Panagiotis Malamos ${ }^{2}$ \\ Maria Douvali' \\ Amalia Ntouraki' \\ Nikos N Markomichelakis \\ '2nd Department of Ophthalmology, \\ Medical School of Athens University, \\ Athens, Greece; ${ }^{2}$ Department of \\ Ophthalmology, NHS, "G Gennimatas" \\ General Hospital, Athens, Greece
}

\begin{abstract}
Aims: To report the anatomic and functional outcomes of intravitreal ranibizumab in idiopathic parafoveal telangiectasia (IPT).
\end{abstract}

Material and methods: Four eyes of three patients were included in this interventional case series. One patient (two eyes) had bilateral IPT (type 2) and two patients (two eyes) had unilateral (type 1) IPT. Retreatment was scheduled in case of leakage persistence in combination with visual acuity (VA) deterioration. Fluorescein angiography and optical coherence tomography were performed together with a full ophthalmic examination at baseline, 1, 3, 6, 9, and 12 months after injection.

Results: One intravitreal injection of ranibizumab was performed in all four eyes. Complete cessation of leakage was documented postintervention in three eyes and partial cessation in one eye, followed by improvement of best corrected VA in one of them. In all eyes, structural changes of the photoreceptor layer were detected in tomography and were responsible for visual loss, which was in most cases, refractory to the applied therapy.

Conclusion: Use of ranibizumab might be efficient in eliminating leakage activity in the macular region in patients with IPT. Nevertheless, improvement in VA was infrequent. Preexisting early photoreceptor alteration in IPT might render such patients unable to improve VA.

Keywords: idiopathic parafoveal telangiectasis, intravitreal ranibizumab, fluorescein angiography, high-definition optical coherence tomography

\section{Introduction}

Idiopathic parafoveal telangiectasias (IPT) were first described as a discrete clinical entity in $1968 .^{1}$ Telangiectasia of the retinal vasculature in one or both eyes localized to the parafoveal region is the hallmark of the disease, accompanied by a late-stage diffuse leakage of the dye in fluorescein angiography located mainly temporal to the fovea. Furthermore, visual loss and deprivation of macular function have been attributed to structural changes affecting neurosensory retina. ${ }^{2,3}$

Since the pathogenesis of IPT remains unclear and no treatment had been proved efficient in the past years, including grid laser photocoagulation, ${ }^{4}$ photodynamic therapy, ${ }^{5}$ and intravitreal steroid application, ${ }^{6}$ this disease raises increasing interest for research in the field of retinal diseases.

Recently, the main role of antivascular endothelial growth factor (anti-VEGF) in the pathogenesis of many retinal diseases has been recognized. Meanwhile, the introduction of anti-VEGF factors in daily clinical practice has brought evolutionary innovations in the treatment of these diseases. ${ }^{7,8}$ Pegaptanib $\left(\right.$ Macugen $^{\circledR}$ ) and ranibizumab (Lucentis ${ }^{\circledR}$ ) have already been approved by the US Food and Drug Administration for the correspondence: Panagiotis Malamos Department of Ophthalmology, NHS, "G Gennimatas"

General Hospital, Athens, Greece

Tel +30 2l 07768423

Fax +30 21 07489454

Email pmalamos@yahoo.com 
treatment of neovascular age-related macular degeneration. Nevertheless, evidence of efficacy of anti-VEGF factors has also been reported in off-label use of bevacizumab (Avastin ${ }^{\circledR}$ ) for treatment of diabetic retinopathy ${ }^{9}$ and vein occlusion related macular edema. ${ }^{10}$ Common events in the pathogenesis of all these diseases are the high levels of $\mathrm{VEGF}^{11}$ and a destabilized blood-retinal barrier with resulting leakage and formation of new vessels. Recent data also support the role of VEGF in the pathogenesis of IPT. ${ }^{12}$

Currently, bevacizumab, pegaptanib, and ranibizumab have been tested in patients with type 2 IPT $^{13-17}$ with controversial results. It is our aim herein to present our findings from intravitreal injection of ranibizumab as a therapeutic modality in patients with IPT.

\section{Materials and methods}

Four eyes of three patients were included in this interventional case series. Two eyes were classified as type 1 IPT and two as type 2 IPT according to specific criteria., ${ }^{3,18}$ Off-label use of the drug and its potential risks and benefits were discussed with all patients. The study was approved by the local ethics committee of Attikon University Hospital of Athens. Informed consent was obtained from every patient, and authors followed the tenets of the Declaration of Helsinki.

A complete ophthalmic examination was performed at the baseline visit, starting with Snellen best corrected visual acuity (BCVA). All patients underwent a routine angiography procedure with intravenous injection of $5 \mathrm{~mL}$ of a $10 \%$ fluorescein solution (Novartis Pharma AG, Bern, Switzerland). Following the injection, images were taken from the early phase (10-20 seconds) to the late phase (10 minutes). A confocal scanning laser ophthalmoscope (Spectralis, Heidelberg Engineering, Dossenheim, Germany) was used.
From each series, one representative early phase angiogram and one from the late phase were selected for analysis. Later, optical coherence tomography (OCT) images from the macular area were obtained. Spectralis OCT belongs to the spectral domain high definition OCT generation, providing an axial resolution of $5 \mu \mathrm{m}$ and a scanning speed of up to 40,000 A-scans/second. Linear or radial scanning modality was centered at the fovea, measuring $6 \mathrm{~mm}$ scan length for each radial scan. Retinal thickness (RT) values were obtained using Spectralis OCT software. This software recognizes the internal limiting membrane as the inner retinal boundary and the outer band of retinal pigment epithelium (RPE) as the outer retinal boundary. ${ }^{19}$ Results were presented as linear graphic curves provided from the software of the device.

Both fluorescein angiograms (FA) and OCT images were evaluated by a retina specialist, and as soon as the diagnosis was set, intravitreal injection of ranibizumab was performed. Exactly the same procedure was followed 1 month after baseline. Re-treatment criteria were the persistence or relapse of leakage combined with visual acuity (VA) deterioration. Postinjection course is presented, with follow-up visits scheduled 1 and 3 months after treatment and in 3-month intervals until 12 months after baseline. Main outcome parameters were BCVA, leakage activity in late phase angiograms, and morphologic changes in OCT images.

\section{Results}

Two patients were males and one was female. Demographic data and BCVA values of the postinjection course are presented in Table 1.

At baseline, before any intervention, all eyes were presenting hyperfluorescent dots in early phase angiograms corresponding to telangiectatic alterations of the perifoveal

Table I Demographic data of patients and visual acuity values during follow-up

\begin{tabular}{|c|c|c|c|c|c|c|c|c|c|c|}
\hline \multirow[t]{2}{*}{ Patients } & \multirow[t]{2}{*}{ Gender } & \multirow[t]{2}{*}{ Age } & \multirow[t]{2}{*}{ Type } & \multirow[t]{2}{*}{ Eye } & \multirow[t]{2}{*}{$\mathbf{N}$} & \multicolumn{5}{|c|}{$\begin{array}{l}\text { BCVA } \\
\text { FA }\end{array}$} \\
\hline & & & & & & Bsl & $I$ & 3 & 6 & 12 \\
\hline \multirow[t]{4}{*}{ I } & Male & 55 & 2 & OD & 1 & 0.05 & 0.05 & 0.05 & 0.05 & 0.05 \\
\hline & & & & & & + & - & - & - & - \\
\hline & & & & OS & I & 0.7 & 0.7 & 0.7 & 0.7 & 0.7 \\
\hline & & & & & & + & - & - & - & - \\
\hline \multirow[t]{2}{*}{2} & Male & 70 & 1 & OS & I & 0.05 & $0.2^{*}$ & 0.3 & 0.3 & 0.2 \\
\hline & & & & & & + & - & - & - & + \\
\hline \multirow[t]{2}{*}{3} & Female & 45 & I & OS & $\mathrm{I}$ & 0.4 & 0.4 & 0.4 & 0.4 & 0.4 \\
\hline & & & & & & + & + & + & + & + \\
\hline
\end{tabular}

Notes: I, I month after baseline; 3, 3 months after baseline; 6, 6 months after baseline; 12, 12 months after baseline; N, number of injections per eye; OD, right eye; OS, left eye; Type, type of IPT lesion (Gass and Blodi criteria); +, leakage in FA; -, absence of leakage in FA. *BCVA improvement was defined an increase in reading performance of at least 2 Snellen lines.

Abbreviations: BCVA, best corrected visual acuity; Bsl, baseline; FA, fluorescein angiography. 


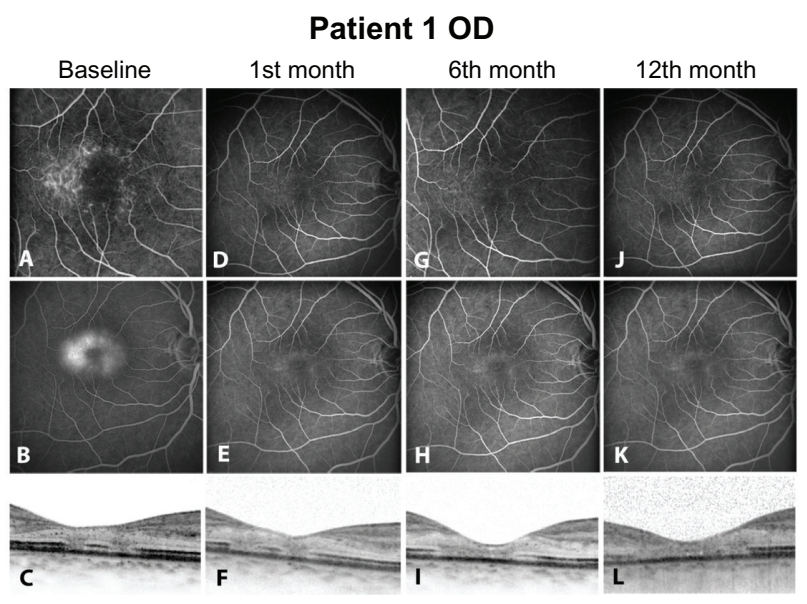

Figure I Right eye of Patient I. Fluorescein angiograms and optical coherence tomography images of each visit are presented. (A, D, G, J) show early phase fluorescein angiograms of baseline and at the Ist, 6th and I2th month. The telangiectatic capillaries in the perifoveal area $(\mathbf{A})$ subside significantly postintervention. (B, E, $\mathbf{H}, \mathbf{K})$ Late phase fluorescein angiograms of the same eye. Leakage/staining in the perifoveal area (B) corresponds to the telangiectatic capillaries of image A. (C, F, I, L) Optical coherence tomography images of each visit detecting severe alterations in photoreceptor layer at inner/outer segments.

capillary network (Figures 1A, 2A, 3A, and 4A). In the late phases, a central hyperfluorescent area surrounding the fovea was manifest, sparing the fovea center (Figures 1B, 2B, $3 \mathrm{~B}$, and $4 \mathrm{~B}$ ). In OCT images, a consistent finding was the structural abnormality at the level of inner/outer segments (IS/OS) of the photoreceptor layer. This layer appears as a thin hyperreflective line overlying the thicker hyperreflective line corresponding to the RPE. In all four eyes, disturbance of the integrity of IS/OS layer was observed (Figures 1C, 2C,

\section{Patient 1 OS}

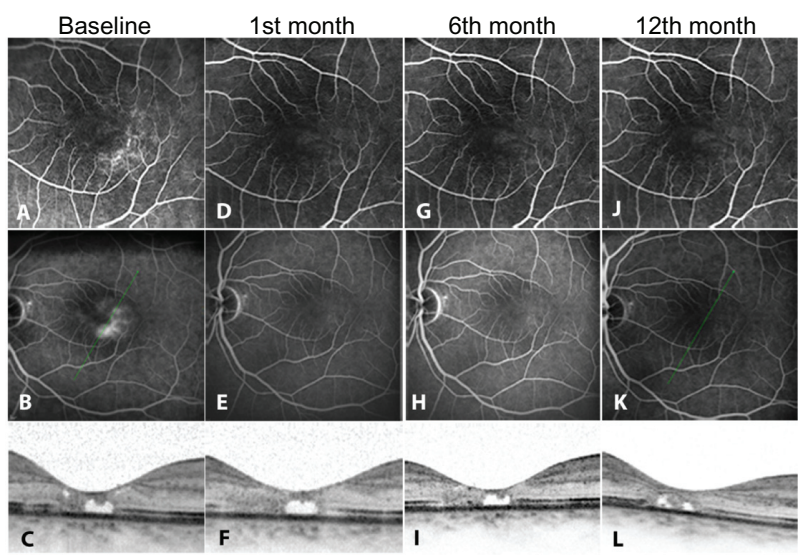

Figure $\mathbf{2}$ Left eye of Patient I. As in the right eye (Figure I), early phase fluorescein angiograms (A, D, G, J) again detect the telangiectatic capillaries of the perifoveal area during follow-up. Leakage/staining in the perifoveal area in late phase (B, baseline) has ceased after treatment $(\mathbf{E}, \mathbf{H}, \mathbf{K})$. Optical coherence tomography images again detect the defects of inner/outer segments. Additionally, a hyporeflective space in the fovea center $(\mathbf{C})$ represents a loss of retinal tissue and remains almost stable throughout the repeated exams $(\mathbf{F}, \mathbf{I}, \mathbf{L})$.
3C, and 4C). Furthermore, hyporeflective cystic spaces were detected in the fovea center in some cases (Figure 2C, 2F, 2I, and $2 \mathrm{~L}$ ). The RT values were within normal limits. Lesions were classified in IPT type 1 and 2 according to the criteria of Gass and Yannuzzi et al. ${ }^{1,3}$ At the second visit, 1 month after ranibizumab injection, reduction of hyperfluorescent dots in early phase angiograms was manifest in all eyes, revealing that the majority of the telangiectatic capillary network in the perifoveal area had already subsided (Figures 1D, 2D, 3D, and 4D). Correspondingly, leakage activity in late phase angiograms was decreased in two eyes (Figures 3E, and 4E) or had almost disappeared from the macular region in the other two eyes compared to baseline (Figures 1E, and 2E). In one of the four eyes, BCVA improved two Snellen lines (shown in Table 1). Since there were no signs of disease activity and BCVA either improved or did not deteriorate, a second injection was not performed.

At the 3 and 6 months, both eyes of patient 1 (Table 1) presented only minimal hyperfluorescence in FA (Figures $1 \mathrm{G}, 1 \mathrm{H}, 2 \mathrm{G}$, and $2 \mathrm{H}$ ). In the other two eyes, FA showed no considerable differentiation compared to images from previous visits (Figures 3G, 3H, 4G, and 4H). BCVA remained stable (0.3) (Table 1 ), and OCT findings such as hypo-reflective cystic spaces in the fovea center and defects in the level of the photoreceptor layer remained stable without significant change.

Twelve months after initial injection, status of leakage activity remains mainly preserved in two eyes (Figures $1 \mathrm{~K}$ and $2 \mathrm{~K}$ ). In FA late phase images, slight increase

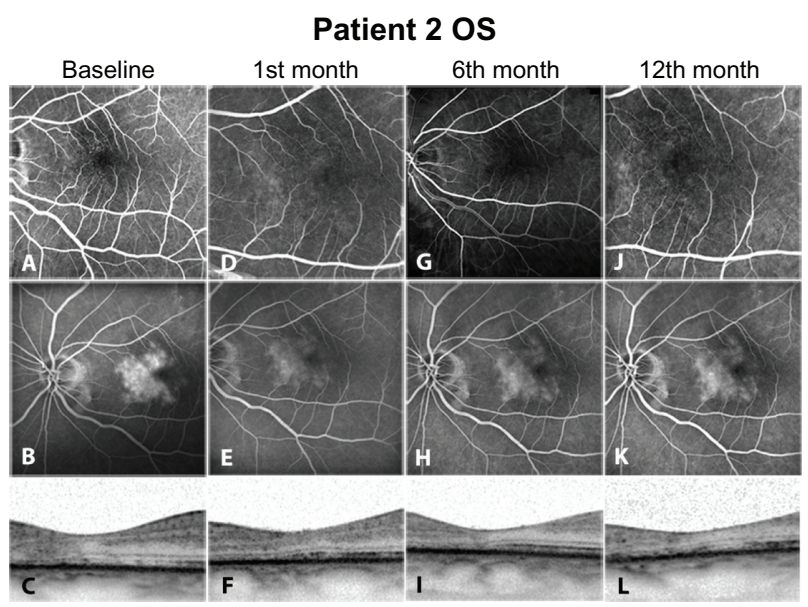

Figure 3 Left eye of Patient 2. Fluorescein angiograms of the early phase (A, D, $\mathbf{G}, \mathbf{J}$ ) show similar findings to those in Figures I and 2. In late phase images, cessation of leakage was documented, as focal hot spots have faded within the entire staining area (B, E, H, K). However, this staining was evident throughout follow-up with slight leakage relapsing by the last visit $(\mathbf{K})$. In optical coherence tomography images, structural changes of the photoreceptors inner/outer segments are a consistent finding (C, F, I, L). 


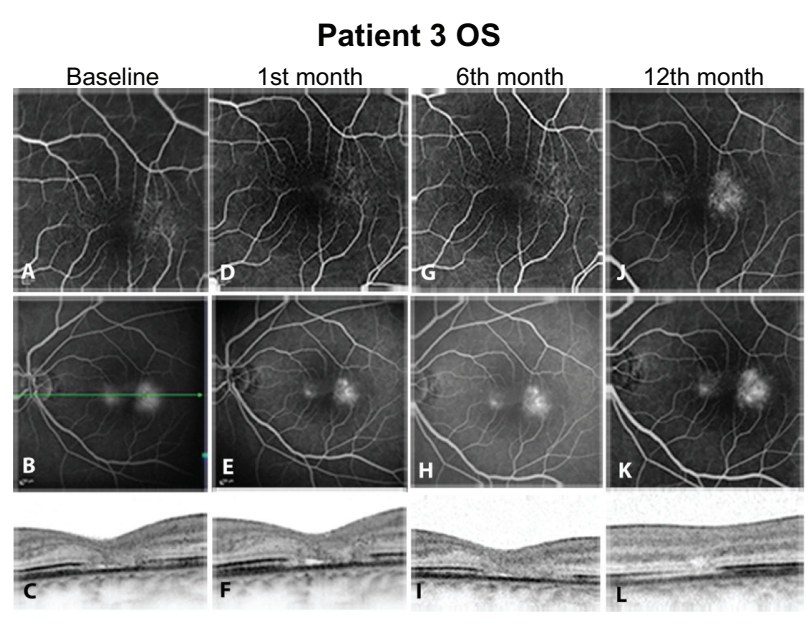

Figure 4 Left eye of Patient 3. Early (A, D, G, J) and late phase (B, E, H, K) Fluorescein angiograms. No cessation of leakage was documented in this case. In optical coherence tomography images, no significant change was documented in the fine retinal anatomy between baseline and posttreatment images. (C, F, I, L) As in all cases, photoreceptor defects were detected at the inner/outer segments level, without significant change in retinal thickness.

of leakage was documented in two cases (Figures $3 \mathrm{~K}$ and $4 \mathrm{~K})$. In OCT images, IS/OS fragmentation seemed to persist (Figure 3L). The BCVA value remained at the same level as in the previous visits (Table 1).

\section{Discussion}

In this study, we present our results from the use of intravitreal ranibizumab in eyes with IPT. There has been reasonable evidence thus far concerning the pathophysiologic mechanism of IPT, which support the use of anti-VEGF factors as a therapeutic option for this discrete entity. Specifically, it is believed that thickening of the wall of retinal capillaries due to proliferation of basement membrane and degeneration of pericytes in type 2 IPT can lead to narrowing of the capillary lumen. ${ }^{20}$ Disturbed exchange of oxygen and substrates between the vascular lumen and neurosensory retina can further induce hypoxia and subsequently increase release of VEGF by retinal cells. Loss of pericytes may render capillaries more susceptible to effects mediated by VEGF. ${ }^{14}$ Vascular leakage and neovascularization are major secondary effects of VEGF activity. ${ }^{18}$

In our cases, ranibizumab managed to extinguish leakage either partially or completely. Complete cessation of leakage was observed in 2 of the 4 eyes postintervention. Partial reduction of leakage was noticed in 1 of the rest of the eyes with residual late staining. In the last case, leakage remained stable after treatment. Correspondingly, telangiectatic alterations of the perifoveal capillaries were not detected anymore in early phase angiograms (Figure 1B-D; Figure 2B-D; Figure 3B-D). Hyperfluorescence in some late phase posttreatment angiograms (Figure 3F-H) could be attributed to staining or atrophy of the RPE, as this was evident in OCT images. The angiographic effect remains 12 months after the injection in two eyes (type 2, bilateral IPT). The other two eyes (type 1, unilateral) presented slight leakage increase at last visit; however, BCVA improved in one of these eyes after treatment. For the rest of the eyes the BCVA remained unchanged from baseline during follow-up.

Despite case 3, it seems that elimination of leakage activity, as evident in FA, may not be a relevant outcome parameter for improvement of visual function in IPT. Lack of visual improvement despite leakage cessation should be reasonably attributed to structural changes of the photoreceptors. Disruption of IS/OS layer has been described and is believed to be a main event in the natural course of IPT. ${ }^{21}$ It is associated with the formation of cystic hyporeflective spaces in the outer retina, while degeneration of neuronal and RPE cells are contributing factors. ${ }^{3}$ Such atrophic changes of the photoreceptors were a common finding in almost all eyes of our study, with accompanying hyporeflective cystic cavities located at the fovea center. It is the opinion of the authors, based on published data, that these cavities represent mainly loss of retinal tissue and are not associated with presence of exudative fluid. ${ }^{22,23}$ This assumption is supported by thickness values in retinas with IPT, which are usually slightly thinner compared to normal retinas. Retinal edema, if present at all, should be minimal, so that thickness values of atrophic retinas return mostly to normal limits, despite evident leakage phenomena. ${ }^{24}$ Thus, status of IS/OS layer should be regarded as a critical factor for visual improvement, and as a response to the applied therapy. Comparing the baseline OCT images of all cases (Figures 1I, 2I, 3I, and 4I), it is evident that in case 3 no neurosensory atrophic cystic changes are present and the IS/OS layer is relatively better preserved than in the rest of the eyes.

Pharmaceutical intervention towards stabilization of the vascular wall and blood-retina barrier in IPT has been investigated in recent studies by other researchers. The efficacy of corticosteroids in stabilizing the blood-retinal barrier and downregulating the production of VEGF is well documented. ${ }^{25}$ Intravitreal triamcinolone acetonide has been proved efficient in reducing late phase angiographic leakage/staining in nonproliferative IPT; however, no visual improvement has accompanied the reduction of angiographic 
phenomena in those cases. ${ }^{6,26,27}$ Similar results have been reported for pegaptanib and bevacizumab concerning their impact in reduction of leakage and telangiectatic alterations. ${ }^{13,14}$ Nevertheless, these anti-VEGF factors have shown additional efficacy so far in improving BCVA performance. Recently, data have been published regarding the use of ranibizumab in IPT, although with controversial results. Monthly use of ranibizumab in nonproliferative IPT type 2 did not reveal a functional benefit after 12 months in two studies. ${ }^{15,16}$ However, in another report of a few IPT type 2 cases, ${ }^{17}$ marked exudation of subretinal fluid with corresponding increase in RT were predominant findings. Ranibizumab managed to eliminate subretinal fluid, decrease RT and leakage, and improve BCVA in all cases. Exudative activity however, if not absent, is usually minimal in IPT type 2, causing only subtle RT changes in OCT as mentioned already. Therefore, exceptional cases with marked subretinal exudation and foveal detachment, contributing to further visual loss, complementary to neurosensory atrophy and photoreceptor disruption, could ideally be eligible for antiVEGF treatment. As in other retinal diseases, rebound effect on parafoveal leakage and central RT at about 3-4 months after treatment imposed the need for repeated injections to achieve a more sustained result. This is one drawback of anti-VEGF treatment in chronic retinal diseases.

Despite encouraging evidence, there are still matters for investigation concerning the use of ranibizumab and other anti-VEGF factors in IPT. Mainly, the safety profile should be thoroughly studied with respect to impact on longterm photoreceptor survival. It has been hypothesized that VEGF plays a nutritional and neuroprotective role for the photoreceptors. ${ }^{27}$ Deprivation of the photoreceptor layer is a discrete primary characteristic of IPT. Long-term use of antiVEGF raises reasonable skepticism about the benefit in VA for such a therapeutic option. Nevertheless, preselection of eyes suitable for intervention should further be investigated. It has been suggested that an active disease stage exists, presenting marked leakage in FA, combined with absence of significant atrophic changes in the neurosensory retina. ${ }^{13}$ It has been noted that more promising posttreatment results in VA performance could be expected in these cases, as well as in cases with exceptionally predominant exudative phenomena. $^{28}$

In conclusion, our case series suggests that the use of ranibizumab in IPT can significantly reduce vascular leakage. Although improvement in VA is not frequent, deterioration of visual function is not documented. Early photoreceptor alteration in IPT, which is mainly asymptomatic, leads to neurosensory atrophy, rendering most of these patients unable to achieve any improvement in VA. Thus, early detection of such structural changes is crucial to providing beneficial treatment to these patients. New spectral domain OCT technology with high-definition imaging of retinal anatomy is an advantageous tool in formulating a diagnosis. Limitations of our study are the small number of patients and lack of control group. Further research with greater series of patients is necessary in order to determine the safety and efficacy of VEGF blockage in IPT.

\section{Disclosure}

The authors report no conflicts of interest in this work. None of the authors has a commercial interest in the materials or methods mentioned, and no financial support was provided. All guidelines for experimental investigations from the local ethics committee were followed. Informed consent was obtained from all participants.

\section{References}

1. Gass JD. A fluorescein angiographic study of macular dysfunction secondary to retinal vascular disease. V. Retinal telangiectasis. Arch Ophthalmol. 1968;80(5):592-605.

2. Gass JD, Oyakawa RT. Idiopathic juxtafoveolar retinal telangiectasis. Arch Ophthalmol. 1982;100(5):769-780.

3. Yannuzzi LA, Bardal AM, Freund KB, Chen KJ, Eandi CM, Blodi B. Idiopathic macular telangiectasia. Arch Ophthalmol. 2006; 124(4):450-460.

4. Park DW, Schatz H, McDonald HR, Johnson RN. Grid laser photocoagulation for macular edema in bilateral juxtafoveal telangiectasis. Ophthalmology. 1997;104(11):1838-1846.

5. De Lahitte GD, Cohen SY, Gaudric A. Lack of apparent short-term benefit of photodynamic therapy in bilateral, acquired, parafoveal telangiectasis without subretinal neovascularization. Am JOphthalmol. 2004;138(5):892-894.

6. Wu L, Evans T, Arévalo JF, et al. Long-term effect of intravitreal triamcinolone in the nonproliferative stage of type II idiopathic parafoveal telangiectasia. Retina. 2008;28(2):314-319.

7. Gragoudas ES, Adamis AP, Cunningham ET Jr, Feinsod M, Guyer DR; for VEGF Inhibition Study in Ocular Neovascularization Clinical Trial Group. Pegaptanib for neovascular age-related macular degeneration. N Engl J Med. 2004;351(27):2805-2816.

8. Rosenfeld PJ, Brown DM, Heier JS, et al; for MARINA Study Group. Ranibizumab for neovascular age-related macular degeneration. $\mathrm{NEngl}$ J Med. 2006;355(14):1419-1431.

9. Avery RL, Pearlman J, Pieramici DJ, et al. Intravitreal bevacizumab (Avastin) in the treatment of proliferative diabetic retinopathy. Ophthalmology. 2006;113(10):1695-1705.

10. Kriechbaum K, Michels S, Prager F, et al. Intravitreal Avastin for macular oedema secondary to retinal vein occlusion: a prospective study. Br J Ophthalmol. 2008;92(4):518-522.

11. Adamis AP, Shima DT. The role of vascular endothelial growth factor in ocular health and disease. Retina. 2005;25(2):111-118.

12. Chew EY. Parafoveal telangiectasis. In: Ryan SJ, Hinton DR, Schachat AP, Wilkinson CP, editors. Retina, 4th ed. St Louis: Mosby; 2006;2:1409-1415.

13. Charbel Issa P, Finger RP, Holz FG, Scholl HP. Eighteen-month follow-up of intravitreal bevacizumab in type 2 idiopathic macular telangiectasia. Br J Ophthalmol. 2008;92(7):941-945. 
14. Vianna RN, Squeri G, Turquetti R, Brasil OF, Burnier MN Jr. Intravitreal pegaptanib reduces fluorescein leakage in idiopathic parafoveal telangiectasis. Can J Ophthalmol. 2008;43(4):492-493.

15. Charbel Issa P, Finger RP, Kruse K, Baumüller S, Scholl HP, Holz FG. Monthly ranibizumab for nonproliferative macular telangiectasia type 2: a 12-month prospective study. Am J Ophthalmol. 2011;151(5): $876-886$.

16. Toy BC, Koo E, Cukras C, Meyerle CB, Chew EY, Wong WT. Treatment of nonneovascular idiopathic macular telangiectasia type 2 with intravitreal ranibizumab: results of a phase II clinical trial. Retina. 2012;32(5):996-1006

17. Lira RP, Silva VB, Cavalcanti TM, de Souza AC, Pinto AP. Intravitreous ranibizumab as treatment for macular telangiectasia type 2. Arch Ophthalmol. 2010;128(8):1075-1078.

18. Gass JD, Blodi BA. Idiopathic juxtafoveolar retinal telangiectasis: update of classification and follow-up study. Ophthalmology. 1993;100(10):1536-1546.

19. Mylonas G, Ahlers C, Malamos P, et al. Comparison of retinal thickness measurements and segmentation performance of four different spectral and time domain OCT devices in neovascular age-related macular degeneration. Br J Ophthalmol. 2009;93(11):1453-1460.

20. Green WR, Quigley HA, De la Cruz Z, Cohen B. Parafoveal retinal telangiectasis: light and electron microscopy studies. Trans Ophthalmol Soc U K. 1980;100(Pt 1):162-170.

21. Sanchez JG, Garcia RA, Wu L, et al. Optical coherence tomography characteristics of group 2A idiopathic parafoveal telangiectasias. Retina. 2007;27(9):1214-1220.
22. Gupta V, Gupta A, Dogra MR, Agarwal A. Optical coherence tomography in group 2A idiopathic juxtafoveolar telangiectasis. Ophthalmic Surg Lasers Imaging. 2005;36(6):482-486.

23. Gaudric A, Ducos de Lahitte G, Cohen SY, Massin P, Haouchine B. Optical coherence tomography in group 2A idiopathic juxtafoveolar retinal telangiectasis. Arch Ophthalmol. 2006;124(10):1410-1419.

24. Charbel Issa P, Holz FG, Scholl HP. Findings in fluorescein angiography and optical coherence tomography after intravitreal bevacizumab in type 2 idiopathic macular telangiectasia. Ophthalmology. 2007;114(9): 1736-1742.

25. Wilson CA, Berkowitz BA, Sato Y, Ando N, Handa JT, de Juan E Jr. Treatment with intravitreal steroid reduces blood-retinal barrier breakdown due to retinal photocoagulation. Arch Ophthalmol. 1992;110(8):1155-1159.

26. Martinez JA. Intravitreal triamcinolone acetonide for bilateral acquired parafoveal telangiectasis. Arch Ophthalmol. 2003;121(11): 1658-1659.

27. Alldredge $\mathrm{CD}$, Garretson BR. Intravitreal triamcinolone for the treatment of idiopathic juxtafoveal telangiectasis. Retina. 2003;23(1):113-116.

28. Nishijima K, Ng YS, Zhong L, et al. Vascular endothelial growth factor-A is a survival factor for retinal neurons and a critical neuroprotectant during the adaptive response to ischemic injury. Am J Pathol. 2007;171(1):53-67.
Clinical Ophthalmology

\section{Publish your work in this journal}

Clinical Ophthalmology is an international, peer-reviewed journal covering all subspecialties within ophthalmology. Key topics include: Optometry; Visual science; Pharmacology and drug therapy in eye diseases; Basic Sciences; Primary and Secondary eye care; Patient Safety and Quality of Care Improvements. This journal is indexed on

\section{Dovepress}

PubMed Central and CAS, and is the official journal of The Society of Clinical Ophthalmology (SCO). The manuscript management system is completely online and includes a very quick and fair peer-review system, which is all easy to use. Visit http://www.dovepress.com/ testimonials.php to read real quotes from published authors. 